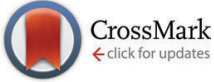

Cite this: Mater. Chem. Front., 2017, 1, 278

Received 13th April 2016, Accepted 17th May 2016

DOI: $10.1039 / \mathrm{c} 6 q m 00012 f$

rsc.li/frontiers-materials

\title{
Nitrogen-doped porous carbon/graphene nanosheets derived from two-dimensional conjugated microporous polymer sandwiches with promising capacitive performance $\dagger$
}

\author{
Kai Yuan, ${ }^{a}$ Ting Hu, ${ }^{\text {bc }}$ Yazhou Xu, ${ }^{\text {b }}$ Robert Graf, ${ }^{d}$ Lei Shi, ${ }^{e}$ Michael Forster, ${ }^{a}$ \\ Thomas Pichler, ${ }^{e}$ Thomas Riedl, ${ }^{c}$ Yiwang Chen $^{* b}$ and Ullrich Scherf*a
}

\begin{abstract}
Conjugated microporous polymers (CMPs) are considered as promising precursors to fabricate multifunctional porous carbons. However, CMPs are formed under kinetic control, and most of them are obtained as amorphous powders without long-range order. Carbon materials derived from CMPs usually preserve the particular structure of the CMP precursors, thus the direct pyrolysis of CMPs into twodimensional (2D) porous carbon nanosheets remains a great challenge. In this work, 4-iodophenylsubstituted graphene (RGO-I) is used both as a building block and a structure directing template for the construction of nitrogen-rich graphene-CMP (GMP) sandwiches using a solution-based approach. The 2D structure of RGO-I with its large aspect ratio allows for the growth of uniform CMP shells onto both sides of the graphene sheets. Thereby, aggregation and restacking of the graphene sheets can be effectively suppressed even during high-temperature treatment. Thereby, well-defined nitrogen-doped porous carbon/ graphene nanosheets were readily obtained by direct pyrolysis of the GMP sandwiches. The sandwich-like nitrogen-doped porous carbon/graphene nanosheets were used as electrode materials for supercapacitor devices with very promising capacitive performance, superior in comparison to the corresponding porous carbons derived from the graphene-free CMPs. The good 2D electron transport ability of graphene together with the intimate interactions between porous carbon and graphene layers provide a combination of large electrochemically active surface area for charge transfer and minimized ion diffusion paths during the charge/discharge process. This unique set of physical properties effectively boosts the capacitive performance values if applied in supercapacitor devices.
\end{abstract}

\section{Introduction}

Supercapacitors are very appealing power sources for various energy storage applications owning to their key advantages, including low cost, rapid charge-discharge kinetics, high power density, superior lifespan and environmental friendliness. ${ }^{1-3}$

\footnotetext{
${ }^{a}$ Bergische Universität Wuppertal, Macromolecular Chemistry Group (buwmakro) and Institute for Polymer Technology, Gauss-Str. 20, D-42119 Wuppertal, Germany.E-mail: scherf@uni-wuppertal.de

${ }^{b}$ College of Chemistry/Institute of Polymers, Nanchang University, 999 Xuefu Avenue, Nanchang 330031, China. E-mail: ywchen@ncu.edu.cn ${ }^{c}$ Bergische Universität Wuppertal, Institute of Electronic Devices, Rainer-Gruenter-Str. 21, 42119 Wuppertal, Germany

${ }^{d}$ Max-Planck-Institut für Polymerforschung, Postfach 3148, D-55021 Mainz, Germany

${ }^{e}$ Electronic Properties of Materials, Faculty of Physics, University of Vienna, Strudlhofgasse 4, A-1090 Vienna, Austria

$\dagger$ Electronic supplementary information (ESI) available: Detailed experimental procedures, FTIR spectra, TGA, CV and GCD profiles, cycling stability and comparison of electrochemical performance. See DOI: 10.1039/c6qm00012f
}

The merits of porous carbon materials are properties such as high chemical stability, lightweight, high specific surface area, good electric conductivity and wide availability, thus enabling them to be the primary candidates for electrode materials for electrical double-layer capacitors (EDLCs). ${ }^{4-8}$ The ion transport time $(\tau)$ is calculated using $\tau=l^{2} / d$, where $l$ and $d$ are the ionic transport distance and coefficient, respectively. ${ }^{9}$ However, the severe pore tortuosity in most available porous carbon materialbased electrodes limits their performance, especially the energy density at high current density due to kinetic limitations in ionic diffusion and transport. ${ }^{10,11}$ Thus, the key to achieve high energy density for porous carbon-based electrodes is to shorten the ion transport time.

In order to address this problem, it is vital to shorten the effective diffusion paths and provide minimized diffusive resistance for maximized mass transport at the electrode/electrolyte interface. As an alternative, nanostructured, two-dimensional (2D) porous carbon materials with their intrinsic large aspect ratios and thinness could significantly shorten the ion transport 
distance in the nanoscale dimension. ${ }^{9,11-16}$ In principle, graphene, with its high theoretical specific surface area $\left(2675 \mathrm{~m}^{2} \mathrm{~g}^{-1}\right)$ and capacitance $\left(550 \mathrm{~F} \mathrm{~g}^{-1}\right.$ ), would be a perfect candidate for elevating the energy density of supercapacitors. ${ }^{17,18}$ Therefore, highly conductive graphene-based 2D materials with large aspect ratio and without pore tortuosity have emerged as promising electrodes for high performance supercapacitors. ${ }^{19-21}$ Unfortunately, the spontaneously occurring graphene layer overlap and aggregation (self-restacking) during electrode processing and electrical cycling, as a result of strong sheet-to-sheet van der Waals interactions, significantly reduce the "real" surface area that is available for charge storage. The stacking process results in a substantial loss of the overall electrochemical performance. ${ }^{9,17,18}$

On the other hand, chemical modification of graphene enables for a fine-tuning of its physical and chemical properties, for example, improving the solution processability in common solvents. ${ }^{22-24}$ Moreover, the covalent functionalization of graphene creates functional groups that can serve as anchor sites for further modification. $^{25-27}$ Thus, ultrathin, functionalized graphene nanosheets with large specific surface area are very promising building blocks for constructing hierarchically organized hybrid architectures. Both sides of the functionalized graphene nanosheets can be utilized for the growth of functional coatings (such as microporous polymer networks) to form unique sandwich-type structures. ${ }^{28,29}$ Such 2D sandwich-type hybrids are potentially attractive materials for application in catalysis, or electrochemical energy conversion and storage. ${ }^{29,30}$

Conjugated microporous polymers (CMPs) are useful precursors for the pyrolytic formation of porous carbons. They show great potential for various applications, e.g., as catalyst carriers or in gas storage and separation devices, supercapacitors and lithium ion batteries. ${ }^{28,29,31-34}$ CMPs are constructed by polymerization of rigid building blocks called tectons. Heteroatoms (such as B, N, P and S) can be controllably introduced into the
CMP networks. The pore size can be defined through the selection of tectons. ${ }^{35-37}$ Consequently, heteroatom-doped porous carbon materials can be obtained by pyrolysis of the CMPs. However, since most of the CMPs are formed under kinetic control, they are mostly obtained as amorphous powders without long-range order. Carbon materials derived from CMPs usually conserve the morphology of the CMP precursors. Therefore, the direct pyrolysis of CMPs into 2D microporous carbon nanosheets with large aspect ratio and high specific surface area remains a challenge.

Herein, we report the functionalization of reduced graphene oxide sheets with 4-iodophenyl functions on both sides of the basal plane. This functionalized graphene then acts both as a building block and a structure-directing template for the construction of nitrogen-rich graphene-CMP (GMP) sandwiches using a solution-based approach. The resulting GMP hybrids possess large aspect ratios and specific BET surface areas of up to $852 \mathrm{~m}^{2} \mathrm{~g}^{-1}$. The microporous polymer shells spontaneously grow onto both sides of the graphene templates, thus guaranteeing a full separation of the individual graphene sheets. So, the sandwich-like architecture effectively prevents the agglomeration of graphene planes during high-temperature conversion into nitrogen-doped porous carbon/graphene nanosheets. The obtained hybrid nanosheets have been used as electrode materials for supercapacitor devices with promising capacitive performance, superior to porous carbon materials originating from the corresponding graphene-free CMPs.

\section{Results and discussion}

Our synthetic approach to graphene-based conjugated microporous polymer (GMP) sandwiches is illustrated in Scheme 1. Reduced graphene oxide (RGO) obtained from the treatment of
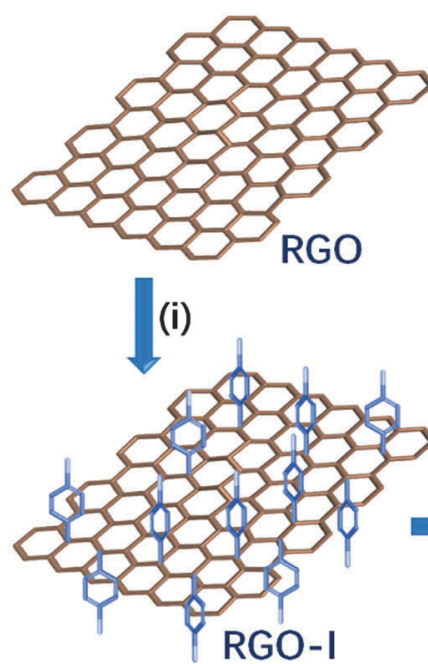

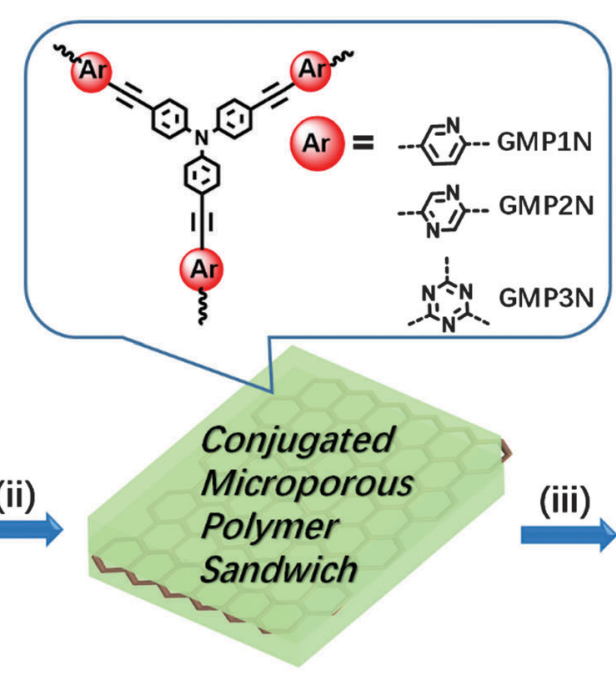

(iii)

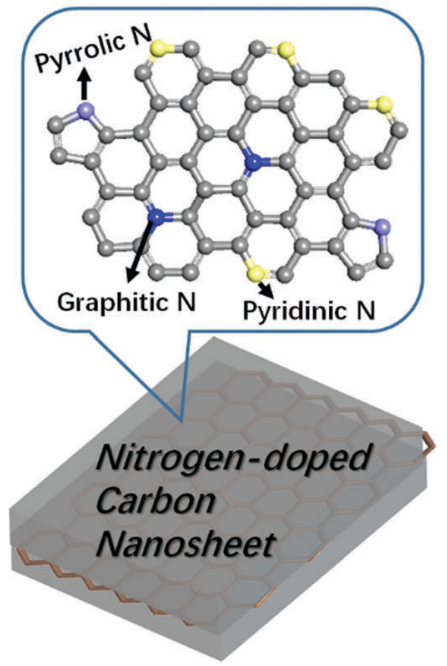

Scheme 1 Preparation of graphene-based conjugated microporous polymer sandwiches and related nitrogen-doped microporous carbon nanosheets. (i) Sodium dodecylbenzenesulfonate, 4-iodophenyl diazonium salt, $0{ }^{\circ} \mathrm{C}(2 \mathrm{~h})$ to RT (4 h); (ii) building blocks: tris(4-ethynylphenyl)amine and 2,5-dibromopyridine, or 2,5-dibromopyrazine, or 2,4,6-trichloro-1,3,5-triazine, argon, $\mathrm{Pd}\left(\mathrm{PPh}_{3}\right)_{4}, \mathrm{Cul}, \mathrm{Et} \mathrm{t}_{3} \mathrm{~N}, \mathrm{DMF}, 120{ }^{\circ} \mathrm{C}, 3$ days; (iii) argon, $\mathrm{RT}$ to $800{ }^{\circ} \mathrm{C}$, $10^{\circ} \mathrm{C} \min ^{-1}, 2 \mathrm{~h}$. 
graphene oxide (GO) with hydrazine hydrate ${ }^{38}$ was modified with 4-iodophenyl diazonium salt under aqueous conditions (Scheme S1, detailed experimental procedures are given in the ESI $\dagger$ ). The iodo functions of the 4-iodophenyl substituents represent good leaving groups $(-\mathrm{I})^{24}$ in the aryl-aryl-couplings, thus favouring polymer growth on the surface of the 4-iodophenylsubstituted graphene (RGO-I) sheets. The RGO-I template is highly dispersible in various organic solvents such as toluene or dimethylformamide (DMF, Fig. S1, ESI $\dagger$ ) without the occurrence of aggregation. Subsequently, covalently crosslinked microporous polymer networks were grown on both sides of the RGO-I nanosheets, resulting in GMP sandwiches. Aryl halide monomers (2,5-dibromopyridine, 2,5-dibromopyrazine or 2,4,6-trichloro1,3,5-triazine) in combination with tris(4-ethynylphenyl)amine were selected as the functional tectons for fabricating the envisaged GMP sandwiches in Sonogashira-Hagihara-type couplings. The precipitated solid materials were collected by filtration and purified by Soxhlet extraction. Finally, the GMP sandwiches were vacuum dried, and denoted as GMP1N, GMP2N and GMP3N for the pyridine-, pyrazine-, and triazinecontaining materials, respectively. For comparison, the corresponding CMPs without RGO-I templates were also prepared by following similar procedures (Scheme S2, ESI $\dagger$ ): the obtained products are named as MP1N, MP2N and MP3N for the pyridine-, pyrazine-, and triazine-containing polymer networks, respectively.

The chemical composition and molecular structure of the GMP sandwiches were studied by Fourier transform infrared (FTIR) spectroscopy and solid-state ${ }^{13} \mathrm{C}$ cross-polarization/magic angle spinning nuclear magnetic resonance (CP/MAS NMR) spectroscopy.
The FTIR spectra clearly indicate the disappearance of the alkynyl $\mathrm{C} \equiv \mathrm{C}-\mathrm{H}$ stretching modes near $3300 \mathrm{~cm}^{-1}$ of the tris(4-ethynylphenyl)amine building blocks as well as of the $\mathrm{C}-\mathrm{Br}$ (ca. $\left.1070 \mathrm{~cm}^{-1}\right)$ or $\mathrm{C}-\mathrm{Cl}\left(\mathrm{ca} .850 \mathrm{~cm}^{-1}\right)$ modes of the aryl halides (Fig. S2, ESI $\dagger$ ). In addition, all GMPs exhibited a typical new $\mathrm{C} \equiv \mathrm{C}$ stretching mode of diarylacetylenes at about $2200 \mathrm{~cm}^{-1}$, thus suggesting the success of the arylacetylene-aryl coupling. Moreover, the ${ }^{13} \mathrm{C}$ NMR spectrum of GMP1N shows typical diaryl-C $\equiv \mathrm{C}$ peak signals in the range of 88-90 ppm (Fig. 1a). The chemical shift at $152 \mathrm{ppm}$ in the ${ }^{13} \mathrm{C}$ NMR spectrum of GMP1N can be assigned to the pyridinic carbons next to the nitrogen atoms (Fig. 1a). The ${ }^{13} \mathrm{C}$ NMR spectra of GMP2N and GMP3N (Fig. S3, ESI $\dagger$ ) can be similarly interpreted indicating the successful formation of the CMP networks on the graphene surfaces. X-ray photoelectron spectroscopy (XPS) investigations (Fig. S4, ESI $\dagger$ ) indicate almost identical components (C, N and O) for all GMPs, as a proof for the structural identity of the GMPs.

The morphology and microstructure of our GMPs were examined by scanning electron microscopy (SEM), transmission electron microscopy (TEM), high resolution TEM (HRTEM) and atomic force microscopy (AFM). All GMPs show similar uniform sheet-like shapes (Fig. 1b-d and Fig. S5, ESI $\dagger$ ), reflecting the morphology of the 2D RGO-I templates (Fig. S1, ESI $\dagger$ ). No "naked" graphene sheets or "free" polymer particles were observed in either SEM or TEM images. These results imply the preference for the formation of hybrids between CMPs and graphene, most of the monomers are deposited on both sides of the graphene sheets to generate sandwich-like objects. The sandwich-like structure also effectively impedes the re-agglomeration of graphene sheets during the following high-temperature treatment. The sizes of these
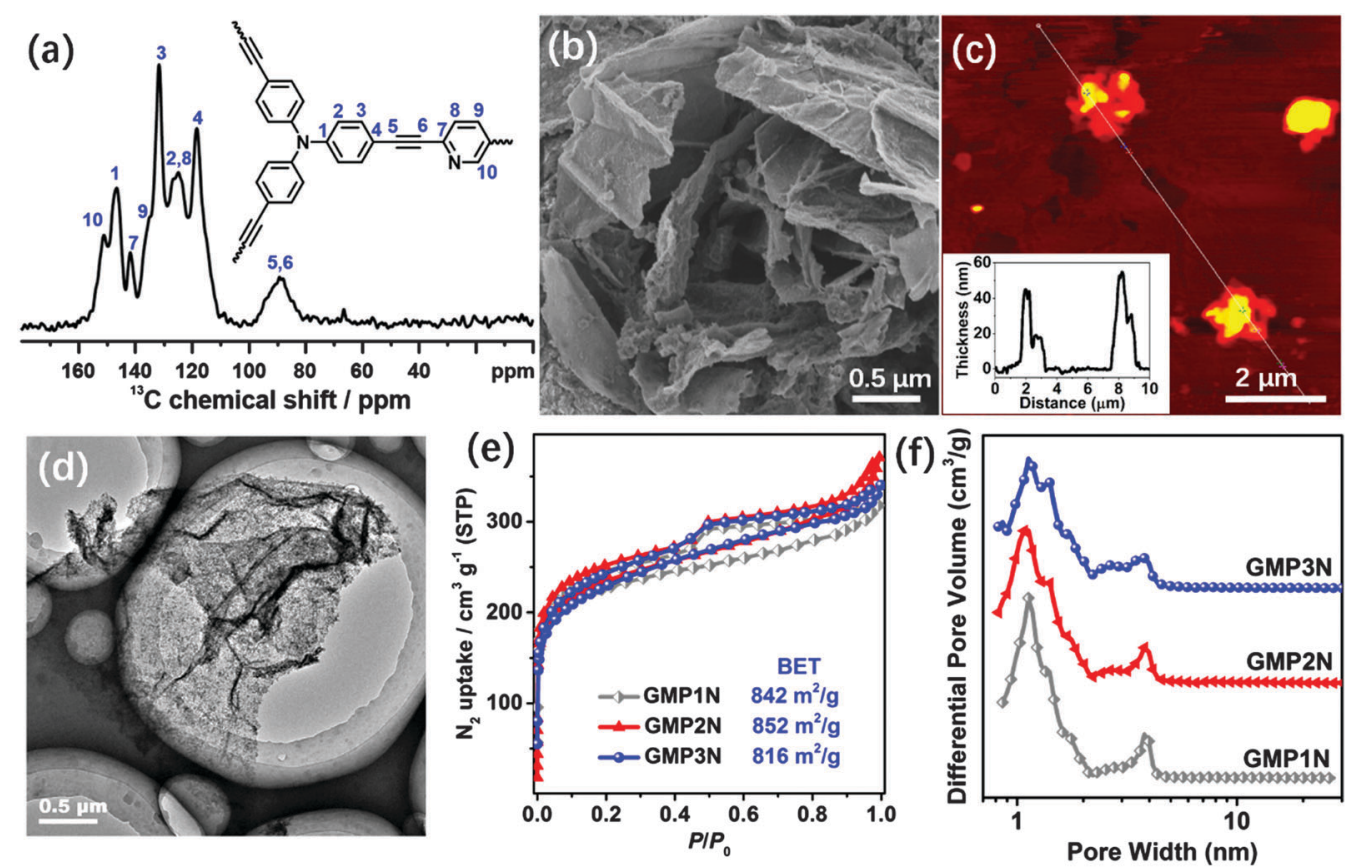

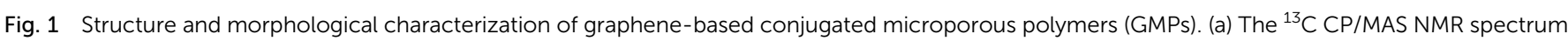
of GMP1N and its peak assignments. (b) SEM, (c) AFM (inset: thickness analyses along the marked line), and (d) TEM images of GMP2N. (e) Nitrogen adsorption/desorption isotherms and (f) pore size distribution profiles of GMPs. 
2D sandwiches range from hundreds of nanometers to several micrometers. The AFM images also illustrate the 2D morphology of the GMPs with a uniform thickness of around $50 \mathrm{~nm}$ (Fig. 1c). As revealed by the TEM images, the wrinkled surface of the nanosheets with numerous dark and light areas (Fig. 1d) indicates the presence of a porous structure with pores of different sizes. Moreover, the micropores that are homogenously distributed over the GMP nanosheets can be directly visualized by HRTEM (Fig. S5, ESI $\dagger$ ). In contrast, CMPs synthesized without RGO-I templates are formed as nanoparticles (Fig. S6, ESI $\dagger$ ). These results confirm that the RGO-I-based template strategy allows for the growth of uniform CMP shells on both sides of the graphene sheets.

The porosity of the GMPs was investigated by $\mathrm{N}_{2}$ adsorption/ desorption measurements performed at $77 \mathrm{~K}$. As indicated in Fig. 1e, all GMPs feature type-IV isotherms, characteristic for a hierarchical pore structure. The steep adsorption increase in the low $p / p_{0}$ region (below 0.1 ) and the hysteretic loops in the high $p / p_{0}$ region reflect the presence of micro- and mesopores, respectively. ${ }^{39}$ This hierarchical distribution of pore sizes is also documented in the plots of the cumulative pore volume $v s$. relative pressure (Fig. S7, ESI $\dagger$ ) as well as in the non-localized density functional theory (NLDFT)-derived pore size distribution diagrams (Fig. 1f). The pore size distribution is of a bimodal shape with maxima at around $1.1 \mathrm{~nm}$ and $3.8 \mathrm{~nm}$ for the three porous GMPs with Brunauer-Emmet-Teller (BET)-related surface areas of 842, 852 and $816 \mathrm{~m}^{2} \mathrm{~g}^{-1}$ (Table S1, ESI $\dagger$ ) for GMP1N, GMP2N and GMP3N, respectively. For comparison, Fig. S8 (ESI $\dagger$ ) presents the sorption isotherms, pore size distributions, and cumulative pore volumes of the graphene-free CMPs. As expected, the graphene-free CMPs exhibit a narrow and unimodal pore size distribution with one maximum centered at around $1.1 \mathrm{~nm}$ with the pore volume and surface area contributions dominatingly from micropores $(<2 \mathrm{~nm})$. In accordance with these findings, the graphene-free CMPs show lower BET surface areas of 739, 632 and $699 \mathrm{~m}^{2} \mathrm{~g}^{-1}$ for MP1N, MP2N and MP3N, respectively.

Microporous, conjugated polymer networks are attractive precursors for the pyrolytic generation of porous, carbonaceous materials. $^{28,29,31,40}$ Thermogravimetric analyses (TGA) suggest that all GMPs are converted into carbon materials with a high yield (ca. 80-90\%) at $800{ }^{\circ} \mathrm{C}$ (Fig. S9, ESI $\dagger$ ). Therefore, we carried out the further carbonizations at $800{ }^{\circ} \mathrm{C}$ for achieving a conversion of the N-rich GMPs into N-doped carbon materials. The resulting $\mathrm{N}$-doped carbon nanosheets derived from GMP1N, GMP2N and GMP3N are denoted as GMP1NC, GMP2NC and GMP3NC, respectively. For comparison, porous carbons were also prepared under similar conditions from the graphene-free CMPs, and are named as MP1NC, MP2NC and MP3NC, respectively. The SEM and TEM images of GMP2NC (Fig. 2a and b) reveal that the obtained carbon material still exhibits a wellpronounced nanosheet morphology. The corresponding HRTEM image (Fig. 2c) shows multilayered, wrinkled graphene-cores that are surrounded by deposits originating from the CMP coatings. The specific surface areas of the obtained hybrid carbon nanosheets were measured by the nitrogen sorption technique at $77 \mathrm{~K}$ (Fig. 2d): the calculated BET surface areas of GMP1NC, GMP2NC and GMP3NC are 523, 642 and $693 \mathrm{~m}^{2} \mathrm{~g}^{-1}$ (Table S2, $\mathrm{ESI} \dagger)$, respectively. The reduction of the BET surface areas for the porous carbon nanosheets compared to the corresponding GMPs is attributed to some restructuring of the initial polymeric networks with a partial shrinkage of the pores during pyrolysis. $^{28,40,41}$ The type-IV isotherms, the cumulated pore volume-plots for different relative pressures (Fig. S10, ESI $\dagger$ )
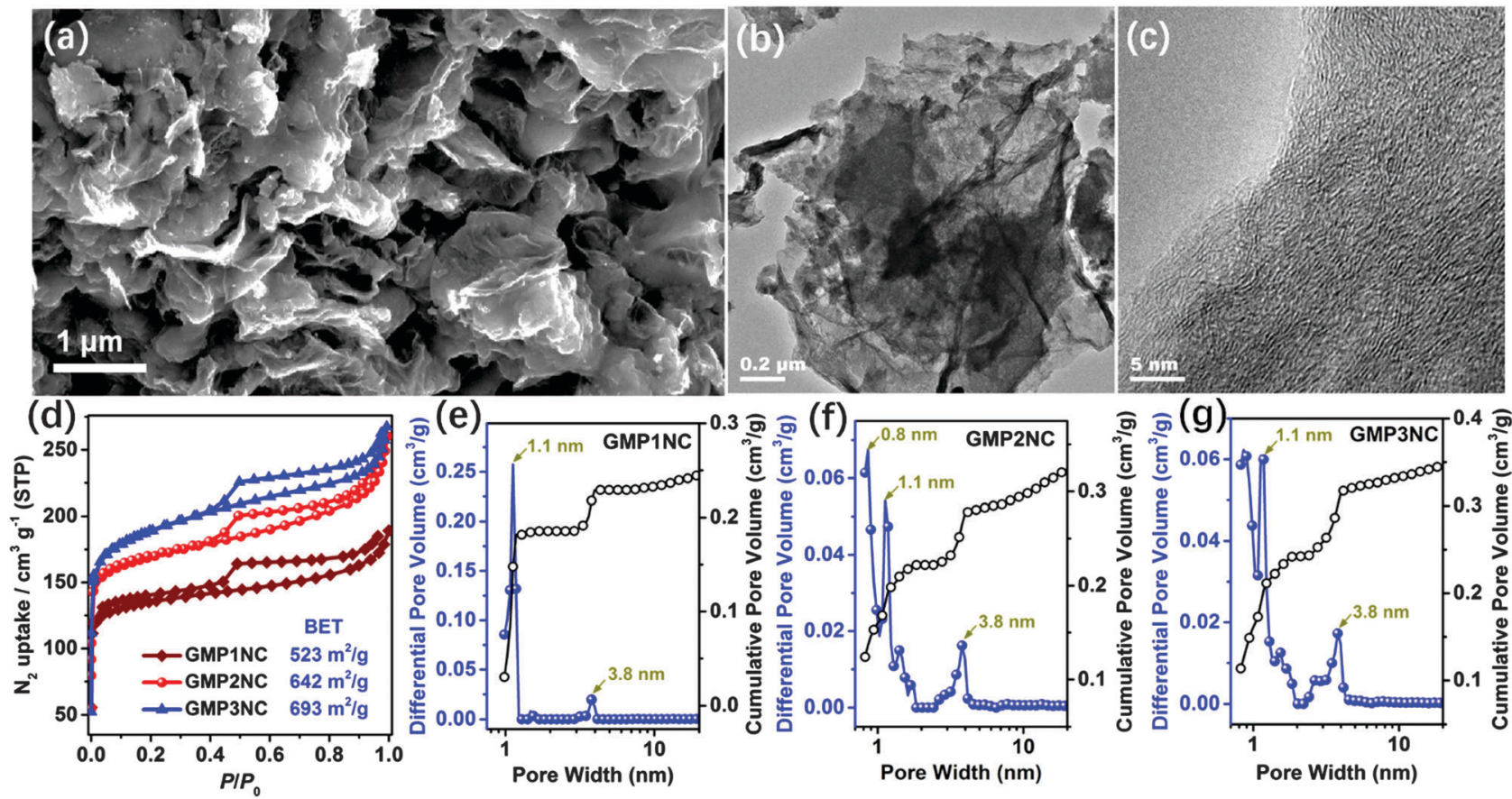

Fig. 2 Morphology and porosity of GMP-derived carbon nanosheets. (a) SEM, (b) TEM and (c) HRTEM images of GMP2N. (d) Nitrogen adsorption/desorption isotherms and $(\mathrm{e}-\mathrm{g})$ NLDFT-based pore size distribution and cumulated pore volume of GMP-derived carbon nanosheets. 
and the NLDFT-based pore size distribution profiles with maxima centered around 1.1 and $3.8 \mathrm{~nm}$ (Fig. 2e and f) suggest the occurrence of a hierarchical porosity with the presence of both micro- and mesopores. In contrast, the isotherms of the graphene-free carbons show a dominating gas adsorption at low $p / p_{0}$ (below 0.1, Fig. S11, ESI $\dagger$ ), which is characteristic of a microporous material. ${ }^{42}$

XPS measurements were carried out for a further investigation of bonding states and compositions of the carbon materials. From our XPS results (Fig. 3a and b and Fig. S12, ESI $\dagger$ ) the atomic percentage of $\mathrm{N}$ in GMP1NC, GMP2NC and GMP3NC is calculated to be about 2.13, 3.05 and $2.61 \mathrm{wt} \%$, respectively. The high resolution XPS $\mathrm{N}$ 1s spectra of GMP1NC, GMP2NC and GMP3NC (Fig. 3a) could be deconvoluted into two subpeaks that have been assigned to pyridinic nitrogens at $398.3 \mathrm{eV}$ and graphitic nitrogens at $400.8 \mathrm{eV}$, respectively. ${ }^{43,44}$ The main sharp peak at $c a .284 .6 \mathrm{eV}$ in the high resolution XPS C 1s spectra for the carbonized samples (Fig. S12, ESI $\dagger$ ) indicates that the majority of carbon atoms are incorporated into graphitic domains. Raman spectroscopy is a versatile characterization tool for carbon materials. ${ }^{45}$ As presented in Fig. 3c, the Raman spectra of GMP1NC, GMP2NC and GMP3NC show relatively strong D bands in relation to the corresponding $\mathrm{G}$ bands $\left(I_{\mathrm{D}} / I_{\mathrm{G}}\right.$ around 1.2). Thereby, the $\mathrm{D}$ bands of the carbon materials are attributed to long range disorder and defects. The introduction of $\mathrm{N}$-dopant heteroatoms as defects also contributes to the intensity of the D band. ${ }^{31}$

Based on the above discussed hierarchically porous structure with well-defined micro- and mesopores, the N-doping and the well-pronounced 2D morphology, the carbon hybrid nanosheets should be promising candidates for application as electrodes for supercapacitors. In this line, we first investigated the electrochemical properties of the porous carbon nanosheets by cyclic voltammetry (CV) and galvanostatic charge-discharge (GCD) techniques using a three-electrode configuration in $6 \mathrm{M}$ aqueous $\mathrm{KOH}$ as electrolyte. Fig. 4a shows the CV curves of the porous carbon nanosheets and the corresponding carbons derived from the graphene-free CMPs at a scan rate of $100 \mathrm{mV} \mathrm{s}^{-1}$ in the potential window of -1 to $0 \mathrm{~V}$. The $\mathrm{CV}$ curves of the porous carbon materials exhibit a rectangular shape, indicating that the charge storage is mainly governed by a typical, capacitive doublelayer behavior. The observed current density and enclosed CV area of the porous carbon nanosheets are higher than those of the corresponding carbons derived from the graphene-free CMPs, thus showing their higher supercapacitive performance. The CV curves of the porous carbon nanosheets at different scan rates from 10 to $200 \mathrm{mV} \mathrm{s}^{-1}$ are shown in Fig. S13 (ESI $\dagger$ ), the current density linearly increases with the scan rate, implying a good electrochemical reversibility. For comparison, the electrochemical properties of RGO, RGO-I and GMPs were also investigated by CV and GCD (Fig. S14, ESI $\dagger$ ). Due to their lower electrical conductivity and hydrophobicity, the GMPs show inferior electrochemical properties compared to the porous carbon nanosheets.

Fig. $4 \mathrm{~b}$ and c present the GCD curves at a current density of $0.2 \mathrm{~A} \mathrm{~g}^{-1}$ and the calculated corresponding specific capacitances. The specific capacitances of GMP1NC, GMP2NC and GMP3NC are 135,273 , and $212 \mathrm{~F} \mathrm{~g}^{-1}$, respectively, at a current density of $0.2 \mathrm{~A} \mathrm{~g}^{-1}$ (Fig. 4c), again higher than those of the carbons derived from the graphene-free CMPs $\left(112,213\right.$, and $184 \mathrm{~F} \mathrm{~g}^{-1}$ for MP1NC, MP2NC and MP3NC, respectively). The GCD profiles and
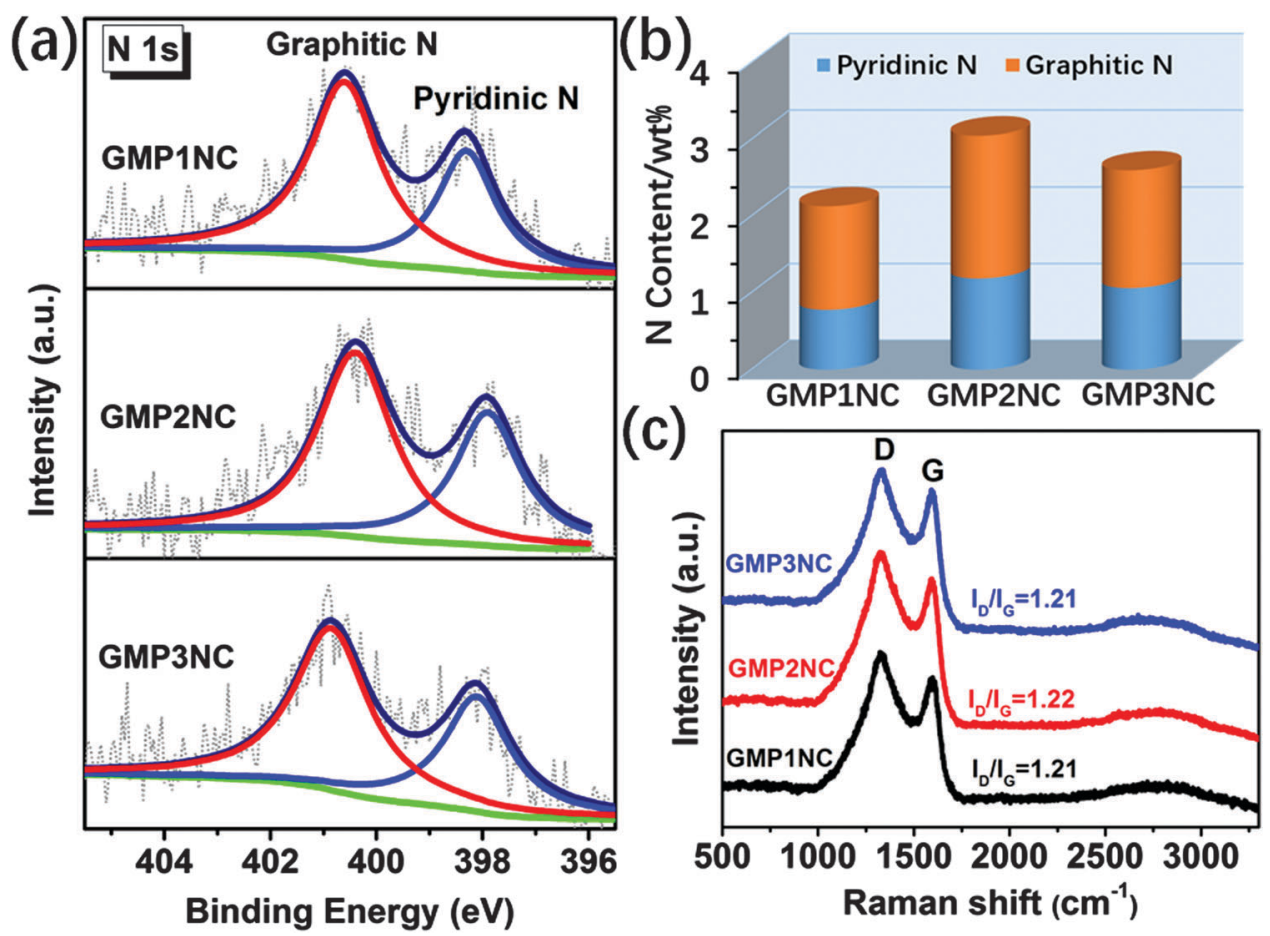

Fig. 3 (a) High resolution N 1s XPS spectra and (b) the content of different nitrogen species in the GMP-derived carbon nanosheets. (c) Raman spectra of GMP-derived carbon nanosheets. 

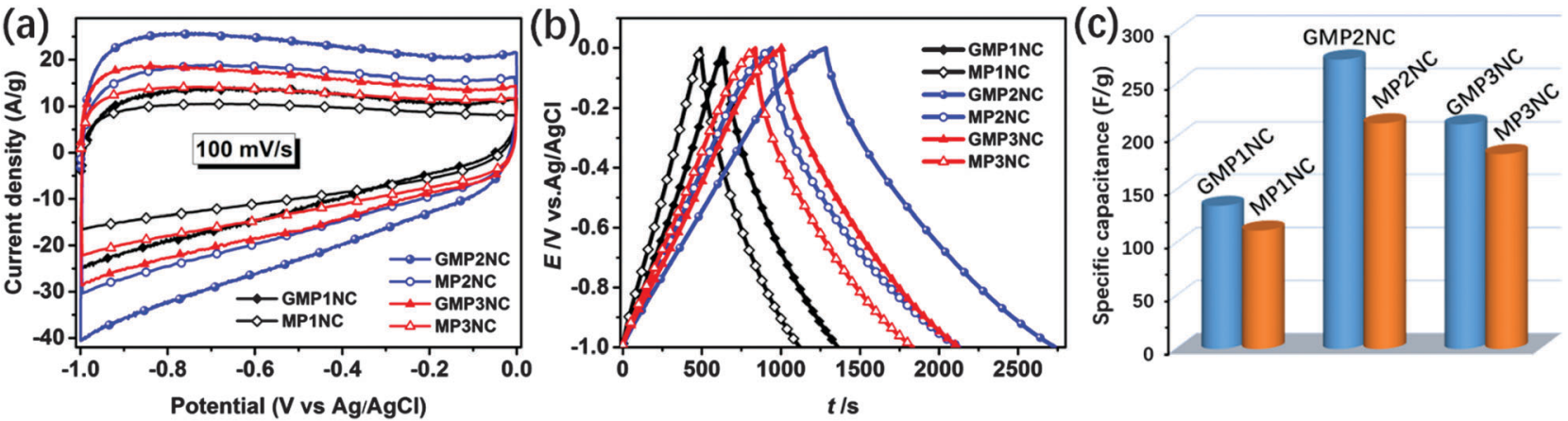

Fig. 4 Electrochemical characterization of the carbon materials derived from GMPs and graphene-free MPs in three-electrode systems using 6 M aqueous $\mathrm{KOH}$ solution as electrolyte. (a) $\mathrm{CV}$ curves at a scan rate of $100 \mathrm{mV} \mathrm{s}^{-1}$. (b) Galvanostatic charge/discharge (GCD) curves and (c) the corresponding gravimetric capacitances at a current density of $0.2 \mathrm{~A} \mathrm{~g}^{-1}$.

corresponding specific capacitances of the porous carbon materials at different current densities are shown in Fig. S15 and S16 (ESI $\dagger$ ). It should be noted that the capacitance retention of the porous carbon nanosheets is significantly higher than those corresponding to carbons derived from the graphene-free CMPs. For example, the calculated specific capacitances of the GMP2NC hybrid are $273 \mathrm{~F} \mathrm{~g}^{-1}$ at $0.2 \mathrm{~A} \mathrm{~g}^{-1}$, and $193 \mathrm{~F} \mathrm{~g}^{-1}$ at $5 \mathrm{~A} \mathrm{~g}^{-1}$, corresponding to a capacitance retention of $70.7 \%$ with the current density increasing from 0.2 to $5 \mathrm{~A} \mathrm{~g}^{-1}$. The obtained capacitance values for graphene-free MP2NC are $213 \mathrm{~F} \mathrm{~g}^{-1}$ at $0.2 \mathrm{~A} \mathrm{~g}^{-1}$, and $105 \mathrm{~F} \mathrm{~g}^{-1}$ at $5 \mathrm{~A} \mathrm{~g}^{-1}$, corresponding to a capacitance retention of $49.3 \%$ with the current density increasing from 0.2 to $5 \mathrm{~A} \mathrm{~g}^{-1}$. Moreover, in a peer comparison, our capacitance values for the GMP2NC nanosheets well compare to those of reduced graphene oxides, N-doped graphene, N-doped carbon and other previously reported carbon materials from the literature (Table S3, ESI $\dagger$ ).

The increased specific capacitance and capacitance retention values of the porous carbon nanosheets should originate from the "implanted" graphene cores of the sandwich-like carbon nanosheets. The charge/discharge process takes advantage of the 2D charge transport ability and superior electrical conductivity of the graphene cores that act as long-distance, in-plane charge transporters and collectors. ${ }^{28,29}$ In synergy with the nitrogendoping, large specific surface areas and the multiscaled porous structure, fast ion and electron transport as well as sufficient space for charge storage are ensured.

To further evaluate the supercapacitive performance of the carbon nanosheets, two-electrode coin-type supercapacitor cells were fabricated using GMP2NC as the active material with $6 \mathrm{M}$ aqueous KOH solution as electrolyte. For comparison, supercapacitors based on graphene-free MP2NC were also fabricated using as similar procedure. The electrochemical performances for the GMP2NC- and MP2NC-based supercapacitors are shown in Fig. 5. Both CV curves exhibit nearly symmetrical rectangular shapes at a scan rate of $10 \mathrm{mV} \mathrm{s}^{-1}$. Upon increasing the scan rate to $200 \mathrm{mV} \mathrm{s}^{-1}$, the GMP2NC-based supercapacitor still maintains a well-pronounced rectangular shape of the CV curve. The enclosed $\mathrm{CV}$ area is distinctly higher than that of the MP2NC-based supercapacitor (Fig. 5a). This difference clearly demonstrates a better supercapacitive behavior for the GMP2NCbased supercapacitor (Fig. S17, ESI $\dagger$ ). ${ }^{43,46}$

Next, GCD measurements were performed at different current densities from 0.2 to $20 \mathrm{~A} \mathrm{~g}^{-1}$ to further characterize the supercapacitor performance. The GCD curves of the GMP2NC-based supercapacitor exhibit high symmetry and linear slopes with a limited voltage $(I R)$ drop even when the current density is increased to $10 \mathrm{~A} \mathrm{~g}^{-1}$, as depicted in Fig. 5b. The GMP2NCbased supercapacitor delivers a gravimetric capacitance value of $73 \mathrm{~F} \mathrm{~g}^{-1}$ (corresponding to $292 \mathrm{~F} \mathrm{~g}^{-1}$ single electrode capacitance), while the MP2NC-based supercapacitor shows a lower gravimetric capacitance of $54.6 \mathrm{~F} \mathrm{~g}^{-1}$ (corresponding to $218.4 \mathrm{~F} \mathrm{~g}^{-1}$ single electrode capacitance) at $0.2 \mathrm{~A} \mathrm{~g}^{-1}$ (Fig. 5c). ${ }^{47}$ At all current densities (0.2-20 A g $\left.{ }^{-1}\right)$, the GMP2NC-based supercapacitor displays smaller $I R$ drops and slower slopes compared to the MP2NCbased supercapacitor (Fig. 5b and Fig. S18, ESI†). These results indicate a higher internal resistance of the MP2NC-based supercapacitor, fully consistent with the aforementioned CV results. Based on this, the area-normalized capacitance values of the GMP2NC-based supercapacitor (e.g. $11.4 \mu \mathrm{F} \mathrm{cm} \mathrm{cm}^{-2}$ at $0.2 \mathrm{~A} \mathrm{~g}^{-1}$ ) are much higher than those of the MP2NC-based supercapacitor (6.2 $\mu \mathrm{F} \mathrm{cm}^{-2}$ at $0.2 \mathrm{~A} \mathrm{~g}^{-1}$ ), as presented in Fig. S19 (ESI $\dagger$ ). The Ragone plots (energy vs. power density) of the GMP2NCand MP2NC-based supercapacitors are shown in Fig. 5d. The energy density of the GMP2NC-based supercapacitor reaches $10.1 \mathrm{~W} \mathrm{~h} \mathrm{~kg}^{-1}$ at a current density of $0.2 \mathrm{~A} \mathrm{~g}^{-1}$ and a power density of $9.5 \mathrm{~kW} \mathrm{~kg}^{-1}$ at a current density of $20 \mathrm{~A} \mathrm{~g}^{-1}$. Notably, the energy density is $33 \%$ higher than that of the MP2N-based supercapacitor $\left(7.58 \mathrm{~W} \mathrm{~h} \mathrm{~kg}^{-1}\right)$ at a current density of $0.2 \mathrm{~A} \mathrm{~g}^{-1}$. In addition, the GMP2NC-based supercapacitor showed excellent cycling stability since $c a .94 .7 \%$ of the initial capacitance is maintained after a long-term charge/discharge cycling test (5000 cycles) at a current density of $5 \mathrm{~A} \mathrm{~g}^{-1}$, see Fig. S20 (ESI $\dagger$ ).

Having demonstrated the excellent performance of a GMP2NCbased supercapacitor, we have finally assembled three individual GMP2NC-based supercapacitors in series to meet specific energy needs for various practical applications. The operating voltage of GMP2NC-based supercapacitors connected in series was measured by $\mathrm{CV}$ at a scan rate of $100 \mathrm{mV} \mathrm{s}^{-1}$ (Fig. 5e). The rectangular $\mathrm{CV}$ curves now display an increased voltage window 
(a)
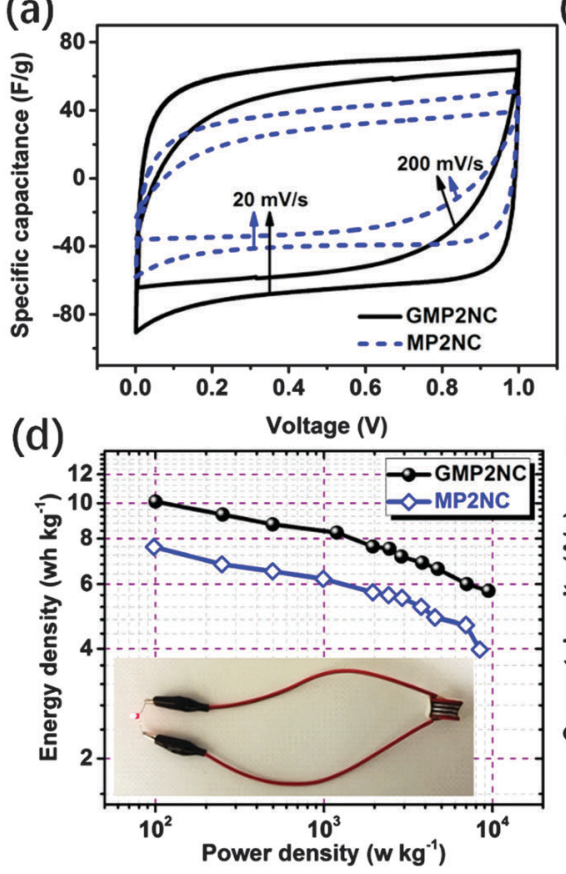

(b)

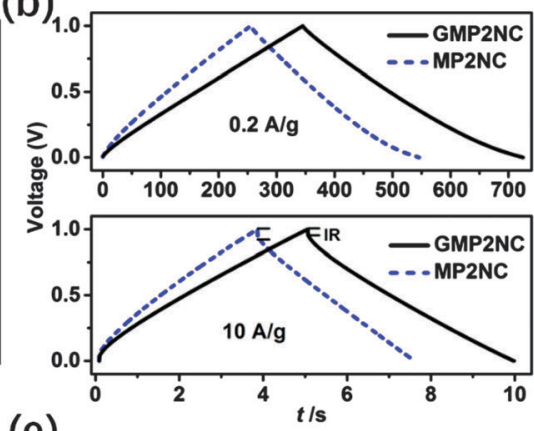

(e)

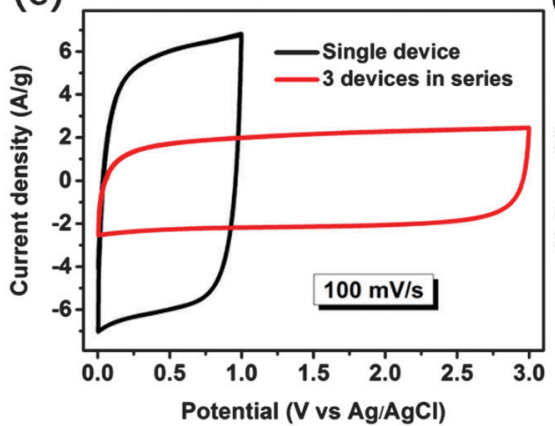

(c)

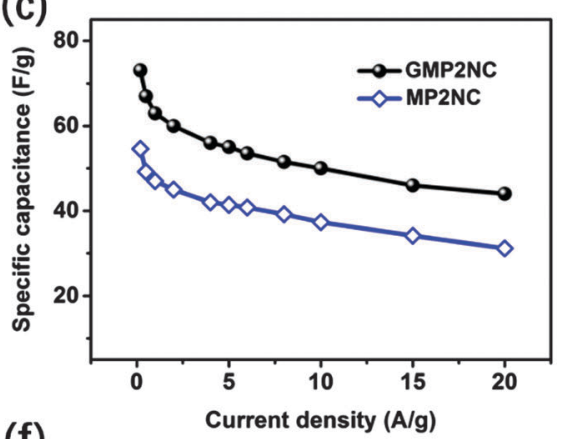

(f)

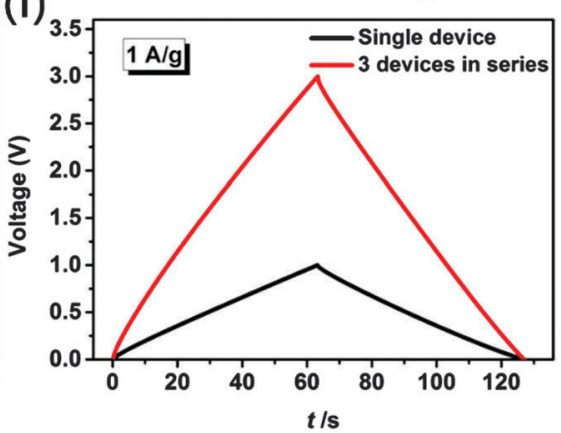

Fig. 5 Electrochemical characterizations of GMP2NC- and MP2NC-based supercapacitor devices using $6 \mathrm{M}$ aqueous KOH solution as electrolyte. (a) CV curves at scan rates of 20 and $200 \mathrm{mV} \mathrm{s}^{-1}$. (b) GCD curves at current densities of 0.2 and $10 \mathrm{~A} \mathrm{~g}^{-1}$. (c) Gravimetric capacitances at different charge-discharge current densities. (d) Ragone plots of gravimetric energy versus power density for GMP2NC-and MP2NC-based supercapacitors, the inset shows the photograph of a red light-emitting-diode (LED) powered by three GMP2NC-based supercapacitor devices connected in series. (e) CV and (f) GCD curves of three GMP2NC-based supercapacitor devices connected in series.

(from $1 \mathrm{~V}$ for a single cell to $3 \mathrm{~V}$ for three GMP2NC-based supercapacitors in series). As expected, the GCD working voltage of the three connected GMP2NC-based supercapacitors is increased by three times with an almost unchanged charge/discharge time compared to the single cell supercapacitor at the same current density (Fig. 5f). The supercapacitor array can be used as a power source when charged at a voltage of $3 \mathrm{~V}$, as exemplified for the lighting of a commercial red light-emitting diode (LED, inset of Fig. 5d). These results confirm the scalability and high potential of GMP2NC-based supercapacitors for energy supply applications.

\section{Conclusions}

In summary, nitrogen-rich CMP-graphene (GMP) sandwiches have been synthesized using a solution-based approach using 4-iodophenyl-modified graphene (RGO-I) both as a building block and a structure-directing template. The controllable growth of uniform CMP shells onto both sides of the graphene sheets effectively prevents re-aggregation and re-stacking of the graphene-based hybrids even during the following hightemperature treatment. Thereby, by direct pyrolysis of the GMP sandwiches well-defined nitrogen-doped porous carbon-graphene nanosheets with a large aspect ratio were obtained. The hierarchically porous nitrogen-doped porous carbon-graphene nanosheets were utilized as electrode materials for supercapacitor devices with promising capacitive performance, superior to the performance of the corresponding porous carbons made from graphene-free CMPs. The good conductivity and the $2 \mathrm{D}$ electron transport ability of the graphene cores of the carbon nanosheets allow for a fast charge transfer during charging/discharging. Moreover, the intimate contact between porous carbon and graphene layers guarantees an optimum interfacial interaction. Together, the optimized electrode/ electrolyte interface and high electrochemically active surface enable a rapid charge transfer and minimized ion diffusion lengths during the charge/discharge processes, thus effectively elevating the capacitive performance of the supercapacitors.

\section{Acknowledgements}

K. Y. would like to thank the fellowship program of the China Scholarship Council for financial support. L. S. and T. P. thank the FWF (NanoBlends I 943-N19) and the EU (FA726006) projects. The authors thank Anke Helfer for performing the TGA analysis, and Sylwia Adamczyk for the AFM measurements.

\section{Notes and references}

1 Y. Gogotsi and P. Simon, Science, 2011, 334, 917-918.

2 P. Simon, Y. Gogotsi and B. Dunn, Science, 2014, 343, 1210-1211.

3 J. Yan, Q. Wang, T. Wei and Z. J. Fan, Adv. Energy Mater., 2014, 4, 1300816.

4 Q. Wang, J. Yan and Z. Fan, Energy Environ. Sci., 2016, 9, 729-762.

5 L. L. Zhang and X. S. Zhao, Chem. Soc. Rev., 2009, 38, $2520-2531$. 
6 P. Simon and Y. Gogotsi, Nat. Mater., 2008, 7, 845-854.

7 Y. Zhai, Y. Dou, D. Zhao, P. F. Fulvio, R. T. Mayes and S. Dai, Adv. Mater., 2011, 23, 4828-4850.

8 T. Lin, I.-W. Chen, F. Liu, C. Yang, H. Bi, F. Xu and F. Huang, Science, 2015, 350, 1508-1513.

9 H. Wang, Z. Xu, A. Kohandehghan, Z. Li, K. Cui, X. Tan, T. J. Stephenson, C. K. King'ondu, C. M. B. Holt, B. C. Olsen, J. K. Tak, D. Harfield, A. O. Anyia and D. Mitlin, ACS Nano, 2013, 7, 5131-5141.

10 X. Zheng, J. Luo, W. Lv, D. W. Wang and Q. H. Yang, Adv. Mater., 2015, 27, 5388-5395.

11 X. M. Fan, C. Yu, J. Yang, Z. Ling, C. Hu, M. D. Zhang and J. S. Qiu, Adv. Energy Mater., 2015, 5, 1401761.

12 J. Hou, C. Cao, F. Idrees and X. Ma, ACS Nano, 2015, 9, 2556-2564.

13 D. Krishnan, K. Raidongia, J. Shao and J. Huang, ACS Nano, 2014, 8, 449-457.

14 M. Sevilla and A. B. Fuertes, ACS Nano, 2014, 8, 5069-5078.

15 Z. Ling, Z. Wang, M. Zhang, C. Yu, G. Wang, Y. Dong, S. Liu, Y. Wang and J. Qiu, Adv. Funct. Mater., 2016, 26, 111-119.

16 Z. Fan, Y. Liu, J. Yan, G. Ning, Q. Wang, T. Wei, L. Zhi and F. Wei, Adv. Energy Mater., 2012, 2, 419-424.

17 R. Raccichini, A. Varzi, S. Passerini and B. Scrosati, Nat. Mater., 2015, 14, 271-279.

18 M. F. El-Kady, V. Strong, S. Dubin and R. B. Kaner, Science, 2012, 335, 1326-1330.

19 F. Bonaccorso, L. Colombo, G. Yu, M. Stoller, V. Tozzini, A. C. Ferrari, R. S. Ruoff and V. Pellegrini, Science, 2015, 347, 1246501.

20 Y. Shao, M. F. El-Kady, L. J. Wang, Q. Zhang, Y. Li, H. Wang, M. F. Mousavi and R. B. Kaner, Chem. Soc. Rev., 2015, 44, 3639-3665.

21 C. Xu, B. Xu, Y. Gu, Z. Xiong, J. Sun and X. S. Zhao, Energy Environ. Sci., 2013, 6, 1388-1414.

22 J. R. Lomeda, C. D. Doyle, D. V. Kosynkin, W.-F. Hwang and J. M. Tour, J. Am. Chem. Soc., 2008, 130, 16201-16206.

23 Y. Zhu, A. L. Higginbotham and J. M. Tour, Chem. Mater., 2009, 21, 5284-5291.

24 K. Yuan, Y. Xu, J. Uihlein, G. Brunklaus, L. Shi, R. Heiderhoff, M. Que, M. Forster, T. Chasse, T. Pichler, T. Riedl, Y. Chen and U. Scherf, Adv. Mater., 2015, 27, 6714-6721.

25 G. Dubey, R. Urcuyo, S. Abb, G. Rinke, M. Burghard, S. Rauschenbach and K. Kern, J. Am. Chem. Soc., 2014, 136, 13482-13485.

26 A. Criado, M. Melchionna, S. Marchesan and M. Prato, Angew. Chem., Int. Ed., 2015, 54, 10734-10750.

27 M. Jahan, Q. Bao and K. P. Loh, J. Am. Chem. Soc., 2012, 134, 6707-6713.
28 X. Zhuang, F. Zhang, D. Wu, N. Forler, H. Liang, M. Wagner, D. Gehrig, M. R. Hansen, F. Laquai and X. Feng, Angew. Chem., Int. Ed., 2013, 52, 9668-9672.

29 X. Zhuang, D. Gehrig, N. Forler, H. Liang, M. Wagner, M. R. Hansen, F. Laquai, F. Zhang and X. Feng, Adv. Mater., 2015, 27, 3789-3796.

30 S. Liu, P. Gordiichuk, Z. S. Wu, Z. Liu, W. Wei, M. Wagner, N. Mohamed-Noriega, D. Wu, Y. Mai, A. Herrmann, K. Mullen and X. Feng, Nat. Commun., 2015, 6, 8817.

31 K. Yuan, X. Zhuang, H. Fu, G. Brunklaus, M. Forster, Y. Chen, X. Feng and U. Scherf, Angew. Chem., Int. Ed., 2016, 55, 6858-6863.

32 Q. Lin, X. Bu, A. Kong, C. Mao, F. Bu and P. Feng, Adv. Mater., 2015, 27, 3431-3436.

33 Y. Z. Liao, J. Weber and C. F. J. Faul, Macromolecules, 2015, 48, 2064-2073.

34 S. N. Talapaneni, T. H. Hwang, S. H. Je, O. Buyukcakir, J. W. Choi and A. Coskun, Angew. Chem., Int. Ed., 2016, 55, 3106-3111.

35 Y. Xu, S. Jin, H. Xu, A. Nagai and D. Jiang, Chem. Soc. Rev., 2013, 42, 8012-8031.

36 A. I. Cooper, Adv. Mater., 2009, 21, 1291-1295.

37 K. Sakaushi and M. Antonietti, Acc. Chem. Res., 2015, 48, 1591-1600.

38 D. Li, M. B. Muller, S. Gilje, R. B. Kaner and G. G. Wallace, Nat. Nanotechnol., 2008, 3, 101-105.

39 R. Du, N. Zhang, H. Xu, N. Mao, W. Duan, J. Wang, Q. Zhao, Z. Liu and J. Zhang, Adv. Mater., 2014, 26, 8053-8058.

40 K. Yuan, P. Guo-Wang, T. Hu, L. Shi, R. Zeng, M. Forster, T. Pichler, Y. Chen and U. Scherf, Chem. Mater., 2015, 27, 7403-7411.

41 X. Zhuang, F. Zhang, D. Wu and X. Feng, Adv. Mater., 2014, 26, 3081-3086.

42 B. D. Assresahegn and D. Belanger, Adv. Funct. Mater., 2015, 25, 6775-6785.

43 J. Zhao, H. Lai, Z. Lyu, Y. Jiang, K. Xie, X. Wang, Q. Wu, L. Yang, Z. Jin, Y. Ma, J. Liu and Z. Hu, Adv. Mater., 2015, 27, 3541-3545.

44 S. Chen, J. Bi, Y. Zhao, L. Yang, C. Zhang, Y. Ma, Q. Wu, X. Wang and Z. Hu, Adv. Mater., 2012, 24, 5593-5597.

45 A. C. Ferrari and D. M. Basko, Nat. Nanotechnol., 2013, 8, 235-246.

46 L. Hao, J. Ning, B. Luo, B. Wang, Y. Zhang, Z. Tang, J. Yang, A. Thomas and L. Zhi, J. Am. Chem. Soc., 2014, 137, 219-225.

47 J. Zhang, J. Jiang, H. Li and X. S. Zhao, Energy Environ. Sci., 2011, 4, 4009-4015. 